\title{
Indirect Sinus Lift: An Overview of Different Techniques
}

\author{
Aruna Wimalarathna* \\ Consultant in Restorative Dentistry, Department of Prosthetic Dentistry, Faculty of Dental Sciences, Sri Lanka
}

*Corresponding author: Aruna Wimalarathna, Consultant in Restorative Dentistry, Department of Prosthetic Dentistry,

Faculty of Dental Sciences, University of Peradeniya, Sri Lanka

\section{ARTICLE INFO}

Received: 幽 February 01, 2021

Published: 蔧 February 08, 2021

Citation: Aruna Wimalarathna. Indirect Sinus Lift: An Overview of Different Techniques. Biomed J Sci \& Tech Res 33(4)2021. BJSTR. MS.ID.005447.

Keywords: Maxillary Sinus; Sinus Lift; Dental Implants; Augmentation; Vertical Bone

Abbreviations: HySiLift: Hydraulic Sinus Lift Technique; $\beta$-TCP: Tricalcium phosphate; PRP: Platelet Rich Plasma

\section{ABSTRACT}

The common physiological changes in edentulous posterior maxilla are the pneumatization of the maxillary sinus and related vertical bone loss. The close proximation of sinus to the crestal bone is a limiting factor for the dental implant placement in the posterior maxilla. Therefore, numerous techniques were implemented to elevate the sinus floor to increase the vertical bone height. The indirect sinus lift is one of the safest and easiest ways of technique that has been introduced in clinical implant dentistry. In this article is expressed an overview of different techniques of indirect sinus lifts.

Clinical Relevance: The general knowledge of different types of indirect sinus lifts will help to utilize them according to the case and the available resources.

Background: To provide an overall summary of different types of indirect sinus lift techniques in one article. The indirect sinus lifts are case sensitive therefore this manuscript will emphasis the indications and limitations of each method when they are applying on patients.

\section{Introduction}

The maxillary sinus (or antrum of Highmore) is a pyramidshaped air-filled space lying within the bilateral maxillae, lateral to the nasal cavity, superior to the maxillary teeth, inferior to the orbital floors, and anterior to the infratemporal fossa. It is present at birth and develops until around the age of 14 years. Maxillary sinuses are the largest among paranasal sinuses [1], with an average of $12.5 \mathrm{~mL}$ in volume [2]. They are lined by a thin bilaminar mucoperiosteal membrane known as the Schneiderian membrane. It comprises with a single-cell osteogenic periosteal layer (cambium layer) on the bone side and ciliated pseudostratified columnar epithelium (respiratory epithelium) on the lumen side. Even though the pneumatization is a poorly understood physiological process, itself causes the expansion of maxillary sinus into the adjacent anatomical structures [3]. In addition, there are some factors such as heredity, craniofacial configuration, nasal mucous membrane pneumatization, sinus surgeries, bone density, air pressure within the sinus and growth hormones may influence the maxillary sinuses pneumatization [4,5]. With the advancement of implant dentistry, the most popular and predictable modality of replacement of missing teeth is dental implants. But, to achieve better osseointegration after placement of an implant there should be good quality and quantity of live bone right around the osteotomy, at least $2 \mathrm{~mm}$ or more [6].

That fundamental requirement may be compromised in edentulous posterior maxilla in the vertical direction due to pneumatization of the maxillary sinus or close proximation of sinus floor to the crestal bone. To overcome that limitation, the sinus lift procedure has been invented in the mid-1970s. There onwards, several techniques and procedures were introduced into the implant dentistry. The rationale behind that all the techniques was an elevation of the sinus membrane to create a sub-antral space for increasing the vertical bone height. Currently, in order to reconstruct the atrophic maxillae, different bone grafting methods are used as autogenous, homogenous and heterogenous grafts, as well as synthetic biomaterials [7]. The accurate diagnosis and a better understanding of bone remodeling at posterior maxilla may be highly valuable for precise dental implant therapy. Therefore, proper patient selection can lead to long the success of the sinus lifting treatments for the deficient posterior maxilla. There are many techniques that are available for sinus lifting. Basically, they can be divided into two broad categories as 
1) The direct method: with lateral antrostomy as a one or two-step procedure and

2) The indirect method: with the osteotome technique with a crestal approach. The indirect sinus lift is also called as subantral sinus augmentation, subcrestal augmentation, sinus floor elevation or transcrestal approach.

Here onwards described the evidence available in literature on different techniques in indirect sinus lift that are used for implant placement in the pneumatized posterior maxilla.

\section{Inflatable Catheter Technique}

The first maxillary sinus lift procedure was performed by Oscar Hilt Tatum Jr, in 1974 [8]. This was followed by placement of two endosteal implants and their restorations. During the year 1975-1979, much of the sinus floor elevation was performed using inflatable catheters. Tatum first presented this novel concept at The Alabama Implant Congress in Birmingham in 1976 and published an article describing the procedure in 1986. Dr Philip Boyne was introduced to the procedure by Tatum in 1977 or 1978. The first publication on the technique was authored by Boyne and James in 1980 when they published case reports of autogenous grafts placed into the sinus and allowed to heal for 6 months, which was followed by the placement of blade implants8. Trans crestal sinus lift using the sinus balloon is a minimally invasive procedure involving few intraoperative complications. Peñarrocha Diago M, et al. [9] revealed that they were able to perform trans crestal sinus lift from $3 \mathrm{~mm}$ of residual bone, gaining a mean height of up to $8.7 \mathrm{~mm}$, and with a $100 \%$ implant success rate one year after prosthetic loading.

\section{Summers Osteotome Technique}

In 1994 Summers introduced the sinus lift technique with the use of osteotomes to elevate the membrane. It was eliminated hammering and making the technique more comfortable for the patient combined with graft material around the implant [10]. This was also known as bone-added osteotome sinus floor elevation technique [11]. This is conceded as a less traumatic and minimally invasive method. The main limiting factors of this technique are the availability of $>5 \mathrm{~mm}$ residual bone height to prevent the membrane perforation and low primary stability of the implant11. On the other hand, this technique was a well-validated surgical option when the residual bone height was $\geq$ to $5-6 \mathrm{~mm}[10,12]$. The survival rate of implants placed simultaneously with indirect sinus lift with bone graft material ranges between $93.5 \%$ and $100 \%$ [13]. Further, the survival rate of the osteotome-installed implants after a mean follow-up time of 3.2- years was $97.4 \%$ in Pjetursson study. There were 3 implants lost before loading and another three were lost in the first and second year. According to the residual bone height, the survival rate was $91.3 \%$ for implant sites with $4 \mathrm{~mm}$ residual bone height, and $90 \%$ for the sites with $4-5 \mathrm{~mm}$ bone height, when compared with that of $100 \%$ in sites with the bone height of above $5 \mathrm{~mm}$. According to implant length, the survival rates were $100 \%$ for $12 \mathrm{~mm}, 98.7 \%$ for $10 \mathrm{~mm}, 98.7 \%$ for $8 \mathrm{~mm}$ and only $47.6 \%$ for $6 \mathrm{~mm}$ implants. This outcome has evidently shown bone height gain $(7.8 \mathrm{~mm} \pm 0.86 \mathrm{~mm})$ which is greater than the average of the osteotome technique $[14,15]$.

\section{Intralift Technique}

Piezoelectric technology is proposed by Torrella et al for the lateral osteotomy surgeries [16]. Based on the use of piezoelectric surgery, sinus lift technique becomes simplified and less intervention as atraumatic as possible. Troedhan and colleagues have developed the Intralif technique to elevate the sinus floor by using piezoelectric surgery based on a specific set of tips for the application of ultrasound. The high-power ultrasonic instruments allow the osteotomies to be made even, in thicker compact cortical bone. The advantage of this system is that it does not cut the soft tissues. Therefore, this surgical instrument can be used to elevate the sinus membrane without perforating it. The piezoelectric surgical sets consist of many different inserts from osteotomies, to diamond-cutting inserts. Immediately after the window is made the sinus membrane is separated from the bone, and a hydropneumatics pressure of the physiologic saline solution is subjected to the piezoelectric cavitation [17]. Vercellotti and colleagues [18] in Italy performed 21 bony window osteotomies by using Piezo surgery System on 15 patients. The inserts were used with a vibration 60$210 \mathrm{~mm}$ with power exceeding 5W. Autogenous bone grafts and platelet-rich plasma were used for all the sinus augmentations in this study. Of the 21 osteotomies, only one resulted in membrane perforation and there was a $95 \%$ success rate.

\section{Hydraulic Sinus Lift Technique}

In this method, the sinus membrane is lifting through a crestal approach, characterized by the hydraulic detachment of the mucosa through injection of a liquid by its spontaneous expulsion or aspiration, and simultaneous filling of the sub Schneiderian space, with solid or semisolid grafting material. But this is involved in prolonging the operating procedure. Furthermore, during this conventional method, a single-use syringe is used which it is not possible to check exactly the progression of the membrane position [12]. In 2013, Andreasi et al. introduce a new method with the advancement of hydraulic pressure exercised on a semisolid graft material to detach the sinus membrane and simultaneously fill the augmented space created this way [19]. This technique is called as HySiLift. There were three components of instruments have used this purpose:

1) a titanium syringe equipped with a micrometric control piston on which it is possible to assemble disposable syringes of various volumes;

2) a surgical steel dispenser available in two forms (conical and cylindrical) and four diameters (two cylindrical of $\varnothing 3.2$ and $4.0 \mathrm{~mm}$ and two conical of $\varnothing 2.8-4.0$ and 3.5-4.6 mm) and; 
3) a needle in surgical steel, with a Luer lock attachment, complementary to that of the single-use syringe.

The single-use syringes can be pre-loaded with the desired amount of graft material that, in our experience, was represented by nanocrystalline hydroxyapatite in an aqueous medium or the syringe containing the graft material as provided by the manufacturer. The tip of the titanium injector is semi-spherical shaped so that to penetrate nearly $3 \mathrm{~mm}$ in the sub-Schneiderian space without damaging the overlying mucosa while the lateral openings allow uniform distribution of the paste-like graft material while forming a dome precisely in correspondence to the future implant site. The threaded portion of the dispenser extends for an about $6 \mathrm{~mm}$ length, thus indicated for ridges of 3-6 mm thickness to ensure the sufficient stability of the tool during the injection maneuver.

\section{Sinu-Lift System}

This is a minimally invasive two-staged indirect sinus lift procedure called a "Sinu-Lift system" that utilizing beta-tricalcium phosphate in conjunction with platelet-rich plasma20. The disposable kit consists of starter drill, curettes, and bone packer. The starter drill ( $\varnothing 3.2-\mathrm{mm}$ ) makes osteotomy towards the sinus membrane which disengages upon contact with the sinus membrane to avoid the rupture. The $3-\mathrm{mm}$ yellow and $4.2 \mathrm{~mm}$ blue curettes are used to gently separate and additional elevation of the membrane. The curettes with colour codings allow the accurate control of the working length providing the desired membrane elevation by minimizing the risk for membrane perforation and post-surgery infections. The bone packer is used to fill the space incrementally with pure phase synthetic $\beta$-TCP (Tricalcium phosphate) sized 500 $1000 \mu \mathrm{m}$ mixed with PRP (Platelet Rich Plasma) was obtained by adding $1 \mathrm{ml}$ Batroxobin and $1 \mathrm{ml}$ of $10 \%$ Calcium gluconate). Mean duration of the procedure was $22.6 \pm 7.5 \mathrm{~min}$ and the mean bone height at the desired area of sinus augmentation was $4.4 \mathrm{~mm}$ which is also statistically significant $(\mathrm{p}<0.01)$. Thus, it is appropriate to conclude that sinus-floor elevation using the "sinu-lift system" is definitely a reliable tool in achieving maximum sinus lift for augmentation [20].

\section{Sinus Augmentation with Simultaneous Implant Placement}

An osteotomy was started with a $2.8 \mathrm{~mm}$ drill to a depth of 3 mm using a stopper and guided with radiographs. The osteotomy was widened with two diameters under drilling off to the desired width (e.g. $3.2 \mathrm{~mm}$ for $4.2 \mathrm{~mm}$; $3.65 \mathrm{~mm}$ for $5 \mathrm{~mm}$ ). The sinus floor was opened by specially designed diamond tips with automatically prevent the Schneiderian membrane penetration. The length of the implant was selected based on the bone height: (a 13-mm for $5 \mathrm{~mm}$ bone, $14.5-\mathrm{mm}$ for $6.5 \mathrm{~mm}$ bone, and a $16-\mathrm{mm}$ for $8 \mathrm{~mm}$ bone). Then the implant was inserted into the osteotomy until it reached the end of the prepared osteotomy and slowly advanced until the sinus floor was penetrated $(<1 \mathrm{~mm})$. A normal saline syringe was connected to the implant via the tubing port. Saline solution (2 cc) was gently injected through the implant and into the sinus. After retracting the saline, the syringe was disconnected from the tubing port and flowable bone graft syringe was then connected. The bone graft material was then slowly injected through the implant into the sinus. After that, the bone graft syringe and the tubing port were disconnected from the implant [21]. Biphasic calcium phosphate in the suspension of a soluble polymer or $\beta$ tricalcium phosphate granulate suspended in a hyaluronic acid matrix were used as injectable bone grafting materials. Then the implant was fully inserted through the osteotomy until the implant aligned with the alveolar crest [21].

According to the study done by Chaushu, et al. [21], the mean initial bone height was $4.21 \pm 0.5$ for the control and $5.44 \pm 0.76$ for the study group $(p<0.01)$. The mean bone gain for the study group was $7.80 \pm 0.5 \mathrm{~mm}$ and $9.3 \pm 0.5 \mathrm{~mm}$ for the control group $(p<0.01)$ and all the implants were placed during the study were osseointegrated at second-stage surgery.

\section{Discussion}

The sinus lifting is a mandatory element in managing atrophic edentulous posterior maxilla. The sinus augmentation procedures have been well established in clinical implant dentistry with many techniques and modifications. In 1980 Boyne and James performed $>10 \mathrm{~mm}$ bone augmentation through lateral approach in the atrophic maxilla with a significant higher post-surgical morbidity and an increased risk of membrane perforation. Therefore, Crestal approach, sinus lift surgery, may be performed with different bone-grafting materials, such as allograft, autogenous bone or heterologous materials, and platelet derivatives themselves or combined with grafting materials, in order to combine the properties of the growth factors that allow a better force control during the sinus floor elevation [12]. Compared to the lateral open approach, the indirect sinus lift technique has many advantages even though it is performed blindly. The advantages are, more conservative, less frequent of sinus membrane rupture, the possibility of simultaneous implantation, good bone healing, better positioning of bone grafting material, no subjected to resorption and high predictable implant survival rate [15]. Further, the 5-year survival rate of implants more to $92.7 \%$ in less than $5 \mathrm{~mm}$ ridge height and $94.9 \%$ for implants placed in more than $5 \mathrm{~mm}$ ridge height after the indirect sinus lift technique [15]. The height bone in between crest of the alveolar bone and the floor of the sinus is the most important factor that influences the survival rate of the implants which placed in sinus augmented sites.

That fact is similarly important for the primary stability of the implants too. According to the literature the amount of available bone directly proportionates to the survival rate. Rosen, et al. [22] concluded that the survival rates are strictly linked to the residual bone height, starting from $96 \%$ when $5 \mathrm{~mm}$ or more of bone is present and dropping it into $85 \%$ when $4 \mathrm{~mm}$ or less bone is 
present. A reduction of the grafted material has been evident over the first 3 months of bone remodeling and remained stable over the whole follow-up period (60 months)15. Regarding the grafting materials, several authors describing a consistent bone formation (6.51 $\mathrm{mm} \pm 2.49 \mathrm{~mm}$ ) even when no grafting material was used after a minimum of 1-year follow-up. On the other hand, some authors have suggested their necessity as the use of a blood clot or platelet concentrates alone may lead to unpredictable results [11]. The autologous bone grafting materials were representing the gold standard, however, might be subjected to extensive resorption and endosinusal contamination due to intraoral pathogens [11]. However, the successful predictability of the indirect sinus lifting procedures are depending on several factors such as the amount of the remaining bone height, primary stability, the ability to elevate the Schneiderian membrane without any tearing, a low membrane detachment force, elasticity and deformation capacity, the amount of elevation, and number of implant insertion sites.

\section{Conclusion}

Gaining additional bone height in atrophic maxilla through sinus augmentation is a well-established clinical procedure in implant dentistry. Compared to the lateral approach technique the vast varieties of indirect sinus lifting techniques are available with more safety and predictable success rates when selecting the cases appropriate to the specific techniques.

\section{Declarations}

\section{Ethical Approval and Consent to Participate}

Not applicable

\section{Consent for Publication}

Not applicable

\section{Availability of Supporting Data}

All the gathered information were presented in the text.

\section{Competing Interests}

The author is declared that there is no conflict of interests on any materials or products which were described in the manuscript.

\section{Funding}

Not applicable

\section{Authors' Contributions}

Single author article

\section{Acknowledgements}

Not applicable

\section{References}

1. Stern A, Green J (2012) Sinus lift procedures: An overview of current techniques. Dent Clin North Am 56(1): 219-233.

2. Gosau M, Rink D, Driemel $O$ (2009) Maxillary sinus anatomy: A cadaveric study with clinical implications. Anat Rec (Hoboken) 292(3): 352-354.

3. Sharan A, Madjar D (2008) Maxillary sinus pneumatization following extractions: A radiographic study. Int J Oral Maxillofac Implants 23(1): 48-56.

4. Shapiro R, Schorr S (1980) A consideration of the systemic factors that influence frontal sinus pneumatization. Invest Radiol 15(3): 191-202.

5. Thomas A, Raman R (1989) A comparative study of the pneumatization of the mastoid air cells and the frontal and maxillary sinuses. AJNR Am J Neuroradiol 10(5): S88.

6. Elian N, Bloom M, Dard M, Cho SC, Trushkowsky RD, et al. (2011) Effect of Interimplant Distance ( 2 and $3 \mathrm{~mm}$ ) on the Height of Interimplant Bone Crest: A Histomorphometric Evaluation. J Periodontol 82(12): 1749-1756.

7. Cardoso CL, Curra C, Santos PL, Rodrigues MFM, Ferreira Junior O, et al. (2016) Current considerations on bone substitutes in maxillary sinus lifting. Rev Clin Periodoncia Implantol Rehabil Oral 9(2): 102-107.

8. https://en.wikipedia.org/wiki/Sinus_lift\#History

9. Peñarrocha Diago M, Galán Gil S, Carrillo García C, Peñarrocha Diago D, Peñarrocha Diago M (2012) Transcrestal sinus lift and implant placement using the sinus balloon technique. Med Oral Patol Oral Cir Bucal 17(1): e122-e128.

10. Summers RB (1994) A new concept in maxillary implant surgery: The osteotome technique. Compendium 15: 152-158.

11. Sathvik N, Nessapan T, Ganapathy D (2019) Indirect sinus lift techniques: A literature review. Drug Invention Today 11(2): 90-93.

12. Emmerich D, Att W, Stappert C (2005) Sinus floor elevation using osteotomes: A systematic review and meta-analysis. J Periodontol 76(8): 1237-1251.

13. Krennmair G, Krainhofer M, Schmid Schwap M, Piehslinger E (2007) Maxillary sinus lift for single implant-supported restorations: A clinical study. Int J Oral Maxillofac Implants 22(3): 351-358.

14. Pjetursson BE, Rast C, Urs Brägger, Schmidlin K, Zwahlen M, et al. (2009) Maxillary sinus floor elevation using the (transalveolar) osteotome technique with or without grafting material. Part I: implant survival and patients' perception. Clin Oral Impl Res 20: 667-676.

15. Lo Giudice G, Iannello G, Terranova A, Lo Giudice R, Pantaleo G, et al. (2015) Transcrestal sinus lift procedure approaching atrophic maxillary ridge: A 60-month clinical and radiological follow-up evaluation. Int J Dent 2015: 1-8.

16. Torrella F, Pitarch J, Cabanes G, Anitua E (1998) Ultrasonic ostectomy for the surgical approach of the maxillary sinus: A technical note. Int J Oral Maxillofac Implants 13(5): 697-700.

17. Troedhan AC, Kurrek A, Wainwright M, Jank S (2010) Hydrodynamic ultrasonic sinus floor elevation an experimental study in sheep. J Oral Maxillofac Surg 68: 1125-1130.

18. Vercellotti T, De Paoli S, Nevins M (2001) The piezoelectric bony window osteotomy and sinus membrane elevation: Introduction of a new technique for simplification of sinus augmentation procedures. Int J Periodontics Restorative Dent 21(6): 561-567.

19. Andreasi B, Lopez MA, Confalone L, Fanali S, Carinci F (2013) Hydraulic sinus lift technique: Description of a clinical case. Ann Oral Maxillofac Surg 1(2): 18 
20. Parthasaradhi T (2015) An alternative maxillary sinus lift technique sinu lift system. J Clin Diagn Res 9(3): ZC33-ZC37.

21. Chaushu L, Chaushu G, Better H, Sarit Naishlos, Roni Kolerman, et al. (2020) Sinus Augmentation with Simultaneous, Non-Submerged, Implant Placement Using a Minimally Invasive Hydraulic Technique. Medicina (Kaunas) 56(2): 75.

\section{ISSN: 2574-1241}

DOI: 10.26717/BJSTR.2021.33.005447

Aruna Wimalarathna. Biomed J Sci \& Tech Res

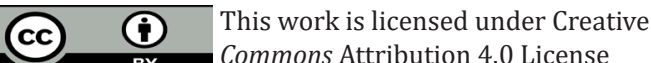

Submission Link: https://biomedres.us/submit-manuscript.php
22. Rosen PS, Summers R, Mellado JR, Salkin LM, Shanaman RH, et al. (1999) The bone-added osteotome sinus floor elevation technique: multicenter retrospective report of consecutively treated patients. Int J Oral Maxillofac Implants 14(6): 853-858.

$\begin{array}{ll}\text { BIOMEDICAL } & \text { Assets of Publishing with us } \\ \text { RESEARCHES } & \text { - Global archiving of articles } \\ \text { - Immediate, unrestricted online access }\end{array}$

